\section{Estratégias de avaliação e melhoria contínua da qualidade no contexto da Atenção Primária à Saúde}

\section{Strategies of assessment and continuous quality improvement in the context of Primary Health Care}

Carlos Eduardo Aguilera Campos 1

1 Núcleo de Estudos em Saúde Coletiva (NESC). Faculdade de Medicina. Universidade Federal do Rio de Janeiro. Praça da Prefeitura da Cidade Universitária. Av. Brigadeiro Trompowski, s. n. Rio de Janeiro, RJ, Brasil. CEP: 21.949-900 E-mail: cadu@vetor.com.br

\begin{abstract}
Recent governmental initiatives allowed for quality assessment proposals to Primary Healthcare in Brazil. This is a new field but a priority in the current strengthening of Family Health strategy following a decade of expansion and consolidation. This article focuses on assessment and quality improvement strategies in the context of Primary Healthcare.

Key words Evaluation, Quality health care, Family health, Family practice
\end{abstract}

\section{Resumo}

Recentes iniciativas governamentais possibilitaram a implantação de propostas de avaliação da qualidade da Atenção Primária à Saúde no Brasil. Este campo até então inexplorado constitui-se uma prioridade no atual processo de fortalecimento da Estratégia de Saúde da Família, após uma década de expansão e consolidação. Este artigo aborda estratégias de avaliação e de melhoria da qualidade no contexto da Atenção Primária em Saúde.

Palavras-chave Avaliação, Qualidade dos cuidados de saúde, Saúde da família, Medicina de família 


\section{Introdução}

A qualidade no âmbito da Atenção Primária à Saúde tornou-se um tema especialmente relevante a partir da reformulação dos sistemas nacionais de saúde nas últimas décadas. A universalidade e a garantia de acesso por meio dos cuidados primários de saúde têm sido preconizadas em vários países do mundo como forma de se alcançar maior equidade e satisfação das expectativas dos usuários. ${ }^{1}$ Entre os diversos aspectos relacionados ao cumprimento desse desafio, também no Brasil, o campo da qualidade no contexto da Atenção Primária em Saúde tem recebido uma atenção especial por parte do Ministério da Saúde, com a constituição de um grupo de trabalho voltado para o seu desenvolvimento. Destaca-se aqui que, no que diz respeito à avaliação de qualidade, essa tem sido a primeira iniciativa que busca uma proposta e uma política de avaliação global da qualidade prestada pelos serviços básicos de saúde no país, 2 nos mesmos moldes de projetos já desenvolvidos em outros países. 3-5

É importante frisar que, anteriormente, algumas secretarias de estado tinham, a partir do pacto da atenção básica, estabelecido parâmetros e critérios de qualidade a embasar propostas de incentivos financeiros aos municípios. 6 Por meio da constituição de um grupo técnico, com a participação municípios e estados, elaborou-se a proposta de "Avaliação para Melhoria da Qualidade da Estratégia Saúde da Família",7 sob a gerência da Coordenação de Acompanhamento e Avaliação do Departamento de Atenção Básica do Ministério da Saúde, e ainda com a cooperação técnica e financeira da Organização Panamericana da Saúde e do Banco Mundial e a assessoria técnica do Instituto de Qualidade em Saúde do Ministério da Saúde de Portugal. Ao envidar esforços para a criação da área de avaliação da qualidade da Atenção Primária à Saúde, o Ministério da Saúde reitera o compromisso de buscar cumprir os princípios da integralidade e da eqüidade das ações e serviços de saúde destinados aos indivíduos, às famílias e à comunidade, anseio de todos os que aspiram por uma condição de vida digna, justa e humana.

Um primeiro instrumento de auto-avaliação da qualidade de gestão, das coordenações e das equipes de saúde da família foi elaborado e disponibilizado aos municípios. Esse instrumento servirá de guia para que os profissionais façam um juízo de valor a respeito do nível de qualidade alcançado no trabalho na Atenção Primária à Saúde. A etapa seguinte, da melhoria da qualidade propriamente dita, é o desafio na busca de consolidar não só o sistema de Atenção Primária à Saúde no país, como a reorganização do modelo assistencial no Brasil, em consonância com os princípios do Sistema Único de Saúde (SUS).

Em que pese o fato de que, no Brasil, o termo usualmente utilizado para designar o sub-sistema de Atenção Primária à Saúde seja denominado de Atenção Básica, utilizamos, neste artigo, para fins de padronização com a terminologia internacional, a expressão Atenção Primária à Saúde (APS). O presente estudo busca abordar as estratégias de avaliação e da melhoria contínua da qualidade no contexto da APS.

\section{A definição da qualidade em atenção primária à saúde}

A qualidade em saúde envolve múltiplos aspectos e pode ser definida como a

"... provisão de serviços acessíveis e eqüitativos, com um nível profissional ótimo, tendo em conta os recursos disponíveis, de forma a alcançar a adesão e a satisfação do usuário" (Hernandez; 1993: 8). 8

Aliada à boa prática do ponto de vista científico e considerando as disponibilidades de recursos existentes, espera-se que os serviços atendam às necessidades de saúde definidas tecnicamente, contemplando o amplo espectro das suas atribuições, quais sejam, a promoção, a prevenção, a assistência e a reabilitação. A atenção à saúde deve buscar otimizar os seus efeitos desejáveis e, por outro lado, minimizar os seus efeitos indesejáveis, para, acima de tudo, responder às expectativas e necessidades manifestas dos usuários. Considera-se ainda a necessidade de analisar o perfil de saúde e doença da comunidade como base para alcançar a redução das desigualdades de acesso e dos riscos de adoecimento entre os indivíduos, famílias e comunidades. ${ }^{9}$

Os critérios utilizados para caracterizar um serviço de qualidade não são definitivos ou absolutos porque a evolução do conhecimento e a dinâmica social estão sempre a exigir mudanças e aprimoramentos na forma como se prestam os serviços. Dependem também de situações muito particulares em que se dá o contexto da assistência e do que os serviços podem produzir, comparativamente à outros em condições semelhantes, em uma dada realidade.

A concepção sobre o que venha ser qualidade depende ainda do lugar que ocupa o sujeito no sistema de saúde. Aqueles responsáveis pela provisão e gestão dos serviços tendem a focalizar a 
sua atenção em determinados aspectos e interesses, tais como rendimento, custo e eficiência. Já no ambiente da prestação dos serviços, os profissionais de saúde têm outras expectativas. De uma forma geral, estão preocupados com a satisfação pessoal, o reconhecimento profissional, a excelência técnica, o acesso à tecnologia, o aprimoramento dos processos individuais e coletivos do cuidado à saúde e um bom ambiente de trabalho, incluindo o conforto, a segurança etc. Por outro lado, o cliente ou usuário entende como qualidade a obtenção dos benefícios esperados diante de demandas, expectativas, carecimentos e necessidades de saúde. A qualidade é um objetivo que vem sendo perseguido por gestores, profissionais e usuários em vários países do mundo, inclusive no Brasil. No caso da Atenção Primária à Saúde, porém, essa é uma questão nova em nossa realidade. A elaboração de normas e critérios a nortear a assistência nesta área nas últimas décadas, esteve circunscrita às áreas técnicas, responsáveis por diversos programas de saúde. Esses podem ser encontrados em publicações sobre as rotinas e procedimentos utilizados para a atenção à saúde integral da mulher, da criança, do combate à tuberculose, a vigilância epidemiológica entre outras. Assim os parâmetros quase sempre foram construídos a partir dos segmentos envolvidos na gestão e na assistência. Também foram propostos de forma segmentada e fragmentada, a atender aspectos particulares da Atenção Primária à Saúde.

Por ocasião da implantação, por parte do Ministério da Saúde, da Estratégia Saúde da Família, foram definidos os princípios operacionais que deveriam ser cumpridos pelos municípios. Entre eles destacam-se: a participação dos gestores, profissionais de saúde, das organizações comunitárias e da população em geral; a aprovação por parte do Conselho Municipal de Saúde; a elaboração de um projeto de implantação; a identificação de áreas prioritárias; a composição das equipes; a cobertura assistencial por equipe de saúde da família; o mapeamento e o diagnóstico das áreas e microáreas de atuação; a implantação e alimentação de sistema de informação; o processo de recrutamento, seleção e contratação dos profissionais; o planejamento local das atividades; a programação das atividades, incluindo um elenco de metas de desempenho e cobertura pré-estabelecidas; a organização de métodos e rotinas de trabalho; a educação permanente. 10 A existência de tais princípios e o monitoramento de seu cumprimento permitiram estabelecer as bases e critérios a serem elegidos para a definição de padrões de qualidade que pudessem ser aplicados nos processos de avaliação. A expansão da Estratégia
Saúde da Família e a progressiva ampliação da cobertura, que alcança, nos dias atuais, cerca de 75 milhões de habitantes, tornam-se um estímulo e um desafio a mais para as iniciativas de avaliação e de melhoria contínua da qualidade.

Conceitos consagrados internacionalmente a respeito das funções da APS nos sistemas de saúde se tornam relevantes para esse debate. São eles a continuidade e o vínculo entre equipes e os usuários, a integralidade da atenção em seus aspectos de coordenação e abrangência dos cuidados prestados, o enfoque familiar e comunitário e a acessibilidade.

Uma APS de qualidade tem sido um requisito bastante enfatizado atualmente em vários países. Esse movimento ganhou força a partir das evidências de que os sistemas nacionais de saúde que priorizam as ações primárias como eixo organizador das práticas sanitárias alcançam melhores indicadores de saúde, menores custos e maior satisfação dos usuários. 11

O sub-sistema da APS deve prover a acessibilidade e aceitabilidade para os seus usuários sem distinção. O acesso depende da estrutura disponível que inclui facilidades para a utilização do serviço, tais como sua distribuição geográfica, localização, planta física, amenidades, tempo de espera, disponibilidade de equipamentos. A tecnologia empregada deve ser adequada, não necessariamente de alta densidade, mas associada a equipes multiprofissionais qualificadas, a uma efetiva organização da assistência, com ênfase no enfoque domiciliar e comunitário. Deve ainda ser capaz de identificar as necessidades de saúde e dar respostas apropriadas. Como parte da estrutura organizacional, inclui-se ainda a existência de registros, de informação e de um sistema de marcação de consultas. A APS qualificada necessita ainda de adequada disponibilidade de recursos financeiros e materiais para atender às necessidades. As expectativas dos usuários relacionadas ao conforto, aos valores humanitários, ao estabelecimento de vínculos de confiança e segurança com a equipe devem ser atendidas.

No campo da qualidade em saúde, trabalha-se com a premissa de que bons processos de trabalho podem levar aos bons resultados. Esses processos incluem a análise da relevância, suficiência ou, por outro lado, da redundância de procedimentos e rotinas relacionados à anamnese, ao exame físico, à investigação laboratorial, ao diagnóstico e ao tratamento, bem como das demais ações realizados na unidade, tais como reuniões de grupo, vacinações, curativos, exames preventivos, visitas domiciliares para a detecção de fatores de risco ou aconselhamento. 
A habilidade dos profissionais em identificar necessidades de saúde e realizar a ação mais apropriada deve ser considerada aos que utilizam o serviço mais também aos que não o demandaram. Assim o maior desafio dos profissionais é identificar o ice-berg, representado pelas doenças e fatores de risco que estão ocultos na comunidade. A isso chama-se necessidades não manifestas ou ocultas. Essas podem ser identificadas por programas que incluam scrennings (teste de glicemia), busca de casos para detectar sintomas ou sinais suspeitos (sintomáticos respiratórios) ou ainda grupos específicos sob algum risco (tabagistas). De toda maneira, a identificação de indivíduos que não buscaram assistência em um dado período de tempo constituise, a priori, um critério de urgência em Atenção Primária à Saúde e deve haver prioridade na sua identificação. 12 A conduta clínica é ainda monitorada em seus aspectos de aderência, conduta e acompanhamento, significando um rol mínimo de ações a ser executado a cada paciente, de acordo com protocolos ou rotinas pré-estabelecidos.

Na prática da Atenção Primária à Saúde muitos dos problemas novos apresentados pelos pacientes são caracterizados por condições agudas, auto-limitadas e, muitas vezes, os agravos relacionados à essas condições se apresentam em um estágio muito precoce. Dessa forma o estabelecimento de diagnósticos bem definidos dos processos patológicos nem sempre são possíveis ou justificados. Esse é o motivo pelo qual são utilizadas classificações de motivos de consultas ou abordagens diagnósticas em termos de sinais e sintomas.

\section{A medida da qualidade na Atenção Primária à Saúde}

Antes que qualquer processo seja considerado satisfatório é necessário relacioná-lo a critérios a respeito do que se considera um atendimento de qualidade. $\mathrm{O}$ estabelecimento desses critérios é a principal tarefa dos estudos de avaliação da qualidade. Esses devem ser adequados à realidade local e devem nortear a busca por padrões mais adequados de assistência. São obtidos mediante pesquisas, mas também por meio da construção de consensos obtidos entre especialistas, considerados experts nas diversas áreas do conhecimento.

Há padrões de qualidade que são consagrados por meio de métodos não experimentais e, como exemplo, àqueles relacionados aos direitos e à dignidade da pessoa humana. Essas condições são definidas socialmente e se relacionam ao que é aceitável e não aceitável do ponto de vista legal e ético. Outro método não-experimental utilizado para definir boas práticas em saúde são os estudos comparativos entre os serviços, tais como número e tipo de prescrições por tipo de condição (prescrição de ansiolíticos em casos de ansiedade), utilização de exames complementares, rotinas e procedimentos utilizadas entre serviços (rotina para detecção de câncer do colo do útero), tendo como parâmetros as diferenças encontradas nos serviços que possam indicar as condições para uma boa prática.

Os estudos experimentais são direcionados à determinar, entre as diversas possibilidades, os processos que alcançam os melhores resultados. São estudos randomizados do tipo caso controle e desenhados para assegurar as diferenças em termos de resultado atribuíveis aos métodos de tratamento em questão (tratamento de varizes por meio de injeção versus cirurgia, acompanhamento de pacientes pósinfarto domiciliar versus hospitalar, utilização de antibióticos versus sinais clínicos pré-determinados de infecções respiratórias).

O uso de traçadores é um outro método utilizado. Tem como base o fato de que determinados eventos, condições ou problema de saúde podem ser utilizados como parâmetro de análise para a qualidade da assistência. Para ser classificado como um traçador, esse tem que ser de fácil identificação, permitir revelar como cada parte do sistema funciona, não isoladamente, mas relacionadas uma às outras. Pressupõe-se que a forma de prover o cuidado rotineiramente determine a ocorrência ou não deste evento, condição ou problema de saúde. Algumas condições são necessárias para a definição de um traçador: seu impacto funcional para os que são afetados por ele; sua prevalência deve ser suficientemente freqüente para permitir a coleta de dados em uma população limitada; sua ocorrência tem que variar de acordo com a utilização e a efetividade da assistência prestada. As técnicas de prevenção e/ou diagnóstico, e/ou tratamento, e/ou reabilitação relacionadas ao traçador tem que estar bem definidas. (surdez associada à infecção do ouvido médio, anemia ferropriva, câncer cervical). A ocorrência dessas condições traçadoras leva à análise do processo de assistência e aos fatores determinantes associados, em termos de falhas na prevenção, diagnóstico, tratamento. 13

$\mathrm{O}$ uso de um conjunto de indicadores relacionado aos casos índice podem também ser utilizados para estabelecer padrões de qualidade. Nesse caso busca-se estabelecer os critérios de boa prática por meio de um score de fatores presentes na assistência de determinada condição ou problema, 
determinando-se, ao final, sua combinação ótima para o alcance da qualidade da assistência (fatores que influenciam a utilização de serviços pelos usuários: custo/conveniência; performance do profissional, comunicação, aderência, atitudes dos pacientes quanto aos profissionais e resolutividade da assistência prestada). ${ }^{14}$

Estudos de resultados geralmente se prestam a determinar as prioridades que devem ser dadas frente aos recursos disponíveis. Resultados não desejados em saúde são classificados classicamente em cinco "ds": death, disease, disability, discomfort e dissatisfaction (morte, doença, disfunção, desconforto e insatisfação). Melhorias organizacionais e de processos podem ser elencados para relacioná-los a bons resultados (benefício do tratamento da hipertensão em termos da diminuição da morbidade e mortalidade por infarto e acidente vascular cerebral; aconselhamento contraceptivo e a ocorrência de aborto; técnicas de educação em saúde e a aderência a determinado tratamento). 15 Entre resultados relevantes em APS devem ser considerados, ainda, a melhoria dos quadros de limitação funcional dos pacientes, que significa valorizar o "cuidado" em lugar da "cura". Essas são situações muito presentes para a análise da assistência aos pacientes crônicos ou idosos. Os resultados dos cuidados incluem ainda a satisfação dos pacientes, a efetividade da comunicação, adesão às recomendações. 16

Conclui-se portanto, que a qualidade na APS abrange aspectos que a difere dos demais níveis do sistema de saúde, como aqueles ligados aos serviços ambulatorial e hospitalar. No contexto da APS, há uma preocupação maior com a identificação das necessidades de saúde dos pacientes independente da busca ao serviço; com o cuidado continuado aos pacientes crônicos ou ainda com a detecção precoce de doenças que podem ser prevenidas em estágios pré-sintomáticos ou sintomáticos, e com a satisfação dos usuários em termos de continuidade, vínculo, adesão e relação com os profissionais de saúde de uma forma geral.

\section{A avaliação e a melhoria da qualidade em Atenção Primária em Saúde}

Uma vez definidos e demarcados os princípios a nortear a qualidade, é necessária a busca de metodologias para se avaliar os sub-sistemas, serviços e equipes envolvidos na Atenção Primária à Saúde. O enfoque mais tradicional utilizado referese àquele proposto por Donabedian, 17 que permite, numa abordagem normativa e focado nos conceitos da administração clássica de eficiência, eficácia e efetividade, definir estratégias, critérios e padrões de medição da qualidade. Nesse sentido, para a medida do nível de qualidade alcançado são comparadas e analisadas as informações a partir das dimensões de estrutura, processo e resultado, a partir de critérios previamente estabelecidos. É importante frisar que, a partir dessa formulação inicial, o referido autor irá propor, posteriormente, uma importante ampliação dos critérios a serem utilizados, os quais ficaram conhecidos como "the seven pillars of quality",18 quais sejam: eficiência, eficácia, efetividade, otimização, aceitabilidade, otimização, legitimidade e eqüidade.

O critério pode ser entendido como uma regra especificando um determinado modo ou curso de ação ou procedimento técnico. Um conjunto de critérios determina uma sequiência lógica de procedimentos referidos a um caso, doença ou fator de risco a ser prevenido. Níveis de pressão diastólica e de colesterol a serem alcançados no controle da hipertensão arterial podem ser exemplos de critérios a serem estabelecidos. Esses norteiam serviços e equipes sobre o modo como deve ser realizada a ação. Para cada um dos critérios ou conjunto deles há padrões referidos como aqueles parâmetros ótimos a serem alcançados. No exemplo acima, o padrão poderia ser a fixação do nível de cobertura de $55 \%$ de pacientes controlados na comunidade para os níveis de pressão diastólica e colesterol. Os padrões podem ser de distintos graus, desde os mínimos indispensáveis até os mais sofisticados e elaborados, considerando um nível avançado de qualidade. Esses são estabelecidos em bases técnicas e científicas a partir da literatura ou por consenso entre os especialistas.

A avaliação segundo um número de critérios que envolvam a estrutura, o processo e o resultado permite, ao final, um juízo de valor sobre o nível de qualidade alcançado, os problemas e as falhas, trazendo a necessidade de buscar estratégias para a sua correção ou a melhoria de aspectos não satisfatórios. A avaliação segue uma seqüência cíclica composta pelas seguintes etapas: entendimento do papel e da missão do serviço no sistema de saúde, estabelecimento dos objetivos da avaliação, escolha dos aspectos ou dimensões a serem avaliadas, construção de critérios, padrões e indicadores; desenho do estudo de avaliação; processo de avaliação; identificação dos problemas e falhas, propostas de mudança; execução das ações de correção e reavaliação. O processo se reinicia indefinidamente em um círculo contínuo.

A avaliação da qualidade não deve ser vista 
como um julgamento a priori, mas uma oportunidade de mudança. Esse é o princípio que norteia análises do tipo "auto-avaliação". A avaliação não deve ser entendida com um fim em si mesmo, mas deve ser acompanhada por propostas que busquem implementar as mudanças. Alguns dos métodos mais utilizados são descritos abaixo.

A Garantia da Qualidade, a Melhoria Contínua da Qualidade e o Monitoramento da Qualidade constituem-se em métodos que buscam estruturar ações que se organizam em um programa interno de qualidade. Eles prevêem o estabelecimento de objetivos bem definidos visando implementar medidas para a garantia e a melhoria da qualidade. São conceitos que envolvem um processo dinâmico e participativo de identificação de problemas e deficiências, definição e implementação de propostas concretas de ação. O ciclo de melhoria de qualidade é um processo contínuo, no qual a avaliação leva ao diagnóstico das deficiências em comparação com parâmetros pré-estabelecidos, a definição de objetivos e metas, a implementação de ações e o retorno à avaliação após um prazo determinado. Esse trabalho de equipe não deve ser encarado nem concebido como um exercício acadêmico ou projeto de pesquisa, mas deve estar adequado para ser desenvolvido como parte integrante da rotina de trabalho. Para isso deve buscar instrumentos e metodologias simples, factíveis e operacionais.

Alguns dos princípios da melhoria contínua da qualidade são: prioridade para a qualidade definida a partir do ponto de vista do cliente; participação de todos os profissionais da equipe, que tem, necessariamente, um papel de sujeitos ativos a desempenhar; ausência de limitações e entraves externos à atuação da equipe; ser uma atividade desenvolvida no âmbito interno do serviço; buscar prevenir os erros por meio de correções com foco prioritário nos processos de trabalho; não ter objetivos finalísticos significando que aquilo que está bom pode ser indefinidamente melhorado; ter o apoio e o compromisso das chefias ou dos níveis hierárquicos superiores da gerência com o esforço das equipes. 19

Para que um programa de garantia ou de melhoria contínua da qualidade cumpra os seus objetivos são necessárias as seguintes condições: provocar mudanças concretas no sentido de um serviço de melhor qualidade; deve-se definir os responsáveis pelas ações; existência de uma decisão superior em proporcionar as condições que não estão ao alcance da equipe, de forma a facilitar a melhoria de qualidade.

Para o monitoramento da qualidade utilizam-se informações previamente existentes nos sistemas de informação, que tenham alimentação sistemática e regular. Os indicadores devem ser escolhidos segundo critérios de relevância para os objetivos do serviço ou para a organização. Podem ser do tipo sentinela. Sua ocorrência é considerada uma falha grave em um dos pontos da cadeia assistencial, impondo uma investigação detalhada (morte materna; internação por complicações de diabetes não controlada; ocorrência de sífilis congênita). ${ }^{20}$ Podem ser utilizados ainda indicadores construídos a partir de dados agregados de desempenho ou cobertura, que permitem avaliar, no tempo, problemas ou insuficiência no alcance de metas. As falhas técnicas da assistência podem ser identificadas e corrigidas por meio desse enfoque (percentual de gestantes captadas no primeiro trimestre da gravidez, percentual de idosos com vacinação em dia). Para esse monitoramento quantitativo podem ser utilizados gráficos ou diagramas de controle, identificando a evolução nos indicadores em intervalos pré-estabelecidos (mensal, semestral, anual). Muitos dos indicadores podem ser construídos a partir do Sistema de Informação da Atenção Básica (SIAB) 21 e utilizados para o monitoramento. As falhas identificadas devem servir para mudanças de estrutura e processos. Outros aspectos importantes que podem ser evidenciados no monitoramento referem-se ao uso desnecessário (uso de antibióticos em casos de gripe, cobertura vacinal acima de $100 \%$ ) ou a subutilização de serviços necessários (cota de exames e medicamentos não utilizados, baixa cobertura de pré-natal; agendas com vagas ociosas para determinado procedimento por tipo de clientela).22 Quanto à eleição de critérios e padrões para a avaliação da qualidade é importante frisar que esses diferem de um país para outro, dependendo da realidade encontrada. Dessa forma métodos e instrumentos utilizados em um país podem ser inadequados para um outro país, considerando o tipo e o estágio em que se encontram os seus sistemas de saúde. Esse é o motivo pelo qual se deve, mesmo com base em diversos instrumentos internacionais, construir uma proposta que reflita a realidade loco-regional em que se esteja atuando.

Por fim, no que se refere à especificidade dos estudos de qualidade aplicados à Atenção Primária à Saúde há que destacar que os modelos usualmente desenvolvidos para a avaliação da qualidade em saúde foram baseados na produção de cuidados hospitalares voltada para o tratamento de doenças. Diversos aspectos relacionados às peculiaridades encontradas na Atenção Primária merecem ser problematizadas. A importância desse tema está no fato de que a qualidade prestada por esse nível de 
assistência presta um papel primordial para que se alcance a qualidade global de todo o sistema. São características próprias deste sub-sistema de atenção aspectos relacionados às suas funções, tais como: o primeiro contato com os problemas do indivíduo e a acessibilidade; a alta incidência de problemas de difícil delimitação; a integração e a coordenação do cuidado com relação aos demais níveis do sistema. Além disso, no que se refere ao trabalho em saúde, tem-se a tônica na promoção e prevenção, na detecção e tratamentos precoces, na atenção de problemas agudos e no controle de processos crônicos. Os componentes psicosociais da atenção são ainda

\section{Referências}

1. Health Council of the Netherlands. European primary care. Draft report presented to the conference Shaping the EU Health Community; 2004 Sep 7-9; The Hague, Netherlands.

2. Felisberto E. Monitoramento e avaliação na atenção básica: novos horizontes. Rev Bras Saúde Matern Infant 2004; 4 : 317-21.

3. The Royal Australian College of General Practitioners. Standards for general ractices. 2nd ed. Melbourne; 2000.

4. The Health Quality Service. The Health Quality Service Accreditation Programme. Standards for primary care trusts. London: Isqua; 2001.

5. The Health Quality Service. The Health Quality Service Accreditation Programme. Standards for primary care teams. 4th ed. London: Isqua; 2001.

6. Ceará. Secretaria de Saúde. Metodologia de melhoria da qualidade em atenção primária à saúde. Fortaleza; 2002.

7. Ministério da Saúde. Avaliação para melhoria da qualidade da Estratégia Saúde da Família. Brasília (DF); 2005.

8. Hernandez PJS. La definición de la calidade de la atención. In: Palomer RM. Garantía de calidad en atención primaria de salud.. Barcelona: Doyma; 1993. p. 7-18. (Monografias Clinicas en Atencion Primaria, n. 13).

9. Donabedian A. The definition of quality and approaches to its management. Explorations in quality assessment and monitoring. Ann Arbor, Michigan: Health Administration; 1982. v. 1

10. Ministério da Saúde. A implantação da Unidade de Saúde da Família. Brasília (DF); 2000.

11. Starfield B. Atenção primária. Equilíbrio entre necessidades de saúde, serviços e tecnologia. Brasília (DF): Unesco, Ministério da Saúde; 2004.

12. IOM (Institute of Medicine). Commitee on the Future of Primary Care. Defining primary care: an interim report. Washington (DC): National Academy; 1994. aspectos a destacar. Todos esses fatores, próprios desse campo de atuação merecem a busca por modelos e ferramentas de avaliação que possam dar conta de buscar a melhoria da qualidade. No momento em que a Estratégia da Saúde da Família alcança um nível de maturidade e consolidação, também aspectos relacionados ao atendimento das expectativas e necessidades dos usuários e dos aspectos ligados ao trabalho interdisciplinar, da capacitação e da educação permanente dos profissionais serão objetos relevantes para a análise da qualidade em futuro próximo. 23

13. Kessner DM, Kalk CE, Singer J. Evaluacion de la calidad de la salud por el Metodo de los Procesos Trazadores. In: OPS (Organización Pan-americana del a Salud). Investigaciones sobre servicios de salud. Washingonton (DC); 1992. p. 555-3. (Serie Publicación Científica, n. 534).

14. Ministério da Saúde. Monitorização da qualidade organizacional dos centros de saúde - MoniQuOr - Instituto de Qualidade em Saúde. 2 ed. Lisboa: Novos Suportes Publicitários; 2004. .

15. Watkins CJ. The measurement of the quality of general ractitioner care. London: The Royal College of General Practitioners; 1989. (Occasional Paper 15).

16. IOM (Institute of Medicine). Community oriented primary care. New directions for health services delivery. Washington (DC): National Academy; 1983.

17. Donabedian. A. La calidad de la atención médica. México (DF): La Prensa Mexicana; 1984.

18. Donabedian. The seven pillars of quality. Arch Pathol Lab Med 1990; 114: 1115-8.

19. Puime A, Otero Hernández PJS, Palomer R. Marquet mejora de la calidad en Atención Primaria. In: Zurro AM. Compendio de atención primaria. Madrid: Harcourt; 2000.

20. Rutstein DD, Berenberg W, Chalmers TC, Child CG, Fishman AP, Perrin EB. Measuring the quality of medical care: a clinical method. N Eng J Med 1976; 294: 582-8.

21. Ministério da Saúde. Secretaria de Assistência à Saúde. SIAB: manual do Sistema de Informação da Atenção Básica. Brasília (DF); 1998.

22. Donabedian A. Twenty years of research on quality of medical care: 1965-1984. Eval Health Prof 1985; 8: 243-65.

23. Sellés JU, Marín. JM. Problemas de calidad en atención primária. In: Palomer RM. Garantía de calidad en atención primaria de salud. Barcelona: Doyma; 1993. p. 19-32. (Monografias Clinicas en Atencion Primaria, n. 13).

Recebido em 25 de maio de 2005

Versão final apresentada em 23 de agosto de 2005

Aprovado em 19 de outubro de 2005 